\title{
Ascorbic acid improves renal microcirculatory oxygenation in a rat model of renal $\mathrm{I} / \mathrm{R}$ injury
}

\author{
Bulent Ergin ${ }^{1}$, Coert J Zuurbier ${ }^{2}$, Rick Bezemer ${ }^{1}$, Asli Kandil ${ }^{3}$, Emre Almac ${ }^{4}$, \\ Cihan Demirci ${ }^{3}$, Can Ince ${ }^{1}$ \\ 'Department of Translational Physiology, Academic Medical Center, University of Amsterdam, Amsterdam, The \\ Netherlands; \\ ${ }^{2}$ Laboratory of Experimental Anesthesiology and Intensive Care, Department of Anesthesiology, Academic \\ Medical Center, University of Amsterdam, The Netherlands; \\ ${ }^{3}$ Department of Biology, Faculty of Science, University of Istanbul, Istanbul, Turkey; \\ ${ }^{4}$ Department of Anesthesiology, St. Antonius Hospital Nieuwegein, Nieuwegein, The Netherlands
}

\section{ABSTRACT}

Background and objectives: Acute kidney injury (AKI) is a clinical condition associated with a degree of morbidity and mortality despite supportive care, and ischemia/reperfusion injury $(\mathrm{I} / \mathrm{R})$ is one of the main causes of AKI. The pathophysiology of $\mathrm{I} / \mathrm{R}$ injury is a complex cascade of events including the release of free oxygen radicals followed by damage to proteins, lipids, mitochondria, and deranged tissue oxygenation. In this study, we investigated whether the antioxidant ascorbic acid would be able to largely prevent oxidative stress and consequently, reduce I/R-related injury to the kidneys in terms of oxygenation, inflammation, and renal failure. Materials and methods: Rats were divided into three groups ( $n=6 /$ group): (1) a time control group; (2) a group subjected to renal ischemia for $60 \mathrm{~min}$ by high aortic occlusion followed by $2 \mathrm{~h}$ of reperfusion (I/R); and (3) a group subjected to I/R and treated with an i.v. $100 \mathrm{mg} / \mathrm{kg}$ bolus ascorbic acid $15 \mathrm{~min}$ before ischemia and continuous infusion of $50 \mathrm{mg} / \mathrm{kg} / \mathrm{hour}$ for $2 \mathrm{~h}$ during reperfusion $(\mathrm{I} / \mathrm{R}+\mathrm{AA})$. We measured renal tissue oxidative stress, microvascular oxygenation, renal oxygen delivery and consumption, and renal expression of inflammatory and injury markers. Results: We demonstrated that aortic clamping and release resulted in increased oxidative stress and inflammation that was associated with a significant fall in systemic and renal hemodynamics and oxygenation parameters. The treatment of ascorbic acid completely abrogated oxidative stress and inflammatory parameters. However, it only partly improved microcirculatory oxygenation and was without any effect on anuria. Conclusion: The ascorbic acid treatment partly improves microcirculatory oxygenation and prevents oxidative stress without restoring urine output in a severe I/R model of AKI.

Address for Correspondence: Dr. Bulent Ergin,

Department of Translational Physiology, Academic Medical Center, University of Amsterdam, Meibergdreef 9, $1105 \mathrm{AZ}$

Amsterdam, The Netherlands

Email: b.ergin@amc.uva.nl

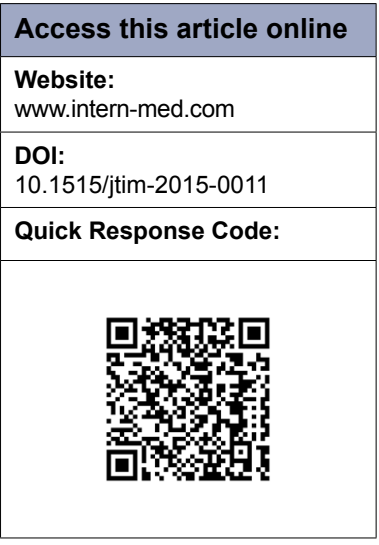

Key words: Ischemia/Reperfusion; ascorbic acid; acute kidney injury; oxygenation; oxidative stress

\section{INTRODUCTION}

Acute kidney injury (AKI) is a critical clinical condition associated with a high degree of morbidity and mortality despite supportive care. Ischemia/ reperfusion injury $(\mathrm{I} / \mathrm{R})$ is one of the main causes of AKI, which is a complex event encountered, for instance, during abdominal aortic surgery ${ }^{[1]}$. Recently, an increasing body of evidence suggests that renal hypoxia contributes to the pathogenesis of $\mathrm{AKI}^{[2-4]}$. It is known that most organs compensate against hypoxia through increases in blood flow or oxygen delivery $\left(\mathrm{DO}_{2}\right)^{[5]}$. Therefore, an increased $\mathrm{DO}_{2}$ may act to improve tissue oxygenation and so attenuates development of hypoxia and injury. The kidney has a unique system and complexity for regulation of blood flow that is dominated by the functional requirements of extracellular fluid and electrolytes load, rather than by local metabolic needs ${ }^{[6]}$. Another way to protect the organ against hypoxia is to reduce oxygen consumption $\left(\mathrm{VO}_{2}\right)$ in 
order to attenuate oxygen requirement. Such a strategy gives rise to decreases in tubular sodium reabsorption ${ }^{[5,7]}$ and glomerular filtration rate (= urine output) in kidney. Abdelkader et al. (2014) documented that even though $\mathrm{I} / \mathrm{R}$ gave rise to reductions in $\mathrm{VO}_{2 \text { ren }}$ and $\mathrm{DO}_{2 \text { ren }}$, renal cortical and inner medullar $\mathrm{PO}_{2}$ (partial oxygen pressure) remained stable ${ }^{[8]}$. Our earlier findings ${ }^{[9,10]}$ indicated that renal $\mathrm{DO}_{2 \text { ren }}$ and $\mathrm{VO}_{2 \text { ren }}$ was reduced after removal of the aortic clamp, and correlated with depletion of the renal microcirculatory oxygenation. This decrease in oxygenation was associated with upregulation of inducible form of nitric oxide synthase (iNOS) and downregulation of endothelial form (eNOS). The relationship between $\mathrm{DO}_{2 \text { ren }}, \mathrm{VO}_{2 \text { ren, }}$ and renal tissue $\mathrm{PO}_{2}$ appears unclear and may vary according to different pathological condition.

The I/ R injury with clinical aortic cross clamping is not limited to the lower extremities, but also causes damage to remote organs and tissues such as lungs, kidneys, heart, and liver ${ }^{[11,12]}$. After the removal of aortic clamp, reperfusion leads to the generation of oxygen-derived free radicals, release of systemic vasoconstrictors, and activation of neutrophils ${ }^{[13]}$. The reactive oxygen species (ROS) is able to damage proteins, lipids, mitochondria, and DNA ${ }^{[14,15]}$. In addition, ROS cause endothelial cell injury and local inflammation, which leads to disturbed microvascular function and consequent tissue hypoxia ${ }^{[16-18]}$. The kidneys are, especially sensitive to this type of injury due to their complex microvascular structure and high oxygen demand ${ }^{[17,19-22]}$.

Several therapeutic agents have been examined to protect the kidneys from I/R injury, including allopurinol, superoxide dismutase, coenzyme $\mathrm{Q}$, antioxidant vitamins, and n-acetylcystein ${ }^{[23,24]}$. L-ascorbic acid (vitamin C) and $\alpha$-tocopherol (vitamin E) also play important roles in the endogenous antioxidant defense systems, and serum levels of these vitamins are significantly decreased in patients with ischemic heart disease ${ }^{[25]}$. In their systematic review, Sadat and co-workers concluded that vitamin $C$ provided effective nephroprotection in patients with contrastinduced AKI, suggesting that ascorbic acid may form part of an effective prophylactic pharmacological regimen ${ }^{[26]}$. Based on the above, it has as yet to be determined which precise mechanism manipulating oxidative stress by vitamin $\mathrm{C}$ affects the injured kidney and if reducing oxidative stress in this way can restore microcirculatory function in terms of oxygen transport and affect renal dysfunction following reperfusion injury. In the present study, therefore, we investigated the hypothesis that using the antioxidant capacity of the ascorbic acid can also efficiently reduce $\mathrm{I} / \mathrm{R}$-induced impairment of the renal oxygenation and renal function following acute $\mathrm{I} / \mathrm{R}$ in the rat kidney.

\section{MATERIALS AND METHODS}

\section{Animals}

All experiments in this study were approved by the Institutional Animal Experimentation Committee of the Academic Medical Center of the University of Amsterdam. Care and handling of the animals were in accordance with the guidelines for Institutional and Animal Care and Use Committees. The study has been carried out in accordance with the Declaration of Helsinki. Experiments were performed on 18 Wistar albino rats (Harlan Netherlands BV, Horst, The Netherlands) with a mean \pm SD body weight of $325 \pm 6 \mathrm{~g}$.

\section{Surgical Preparation}

All animals were anesthetized with an intraperitoneal injection of a mixture of $90 \mathrm{mg} / \mathrm{kg}$ ketamine $\left(\mathrm{Nimatek}^{\circledR}\right.$, Eurovet, Bladel, The Netherlands), $0.5 \mathrm{mg} / \mathrm{kg}$ dexmedetomidine (Dexdomitor, Pfizer Animal Health BV, Capelleaan den IJssel, The Netherlands), and $0.05 \mathrm{mg} / \mathrm{kg}$ atropine sulfate (Centrafarm Pharmaceuticals BV, Etten-Leur, The Netherlands) ${ }^{[27]}$. After preparing a tracheotomy, the animals were mechanically ventilated with $\mathrm{FiO}_{2}$ of 0.4 . Body temperature was maintained at $37 \pm 0.5^{\circ} \mathrm{C}$ during the entire experiment by an external thermal heating pad. Ventilator settings were adjusted to maintain end-tidal $\mathrm{pCO}_{2}$ between 30 and $35 \mathrm{mmHg}$ and arterial $\mathrm{pCO}_{2}$ between 35 and $40 \mathrm{mmHg}$.

For drug and fluid administration and hemodynamic monitoring, vessels were cannulated with polyethylene catheters with an outer diameter of $0.9 \mathrm{~mm}$ (Braun, Melsungen, Germany). A catheter in the right carotid artery was connected to a pressure transducer to monitor mean arterial blood pressure (MAP) and heart rate. The right jugular vein was cannulated for continuous infusion of Ringer's Lactate (Baxter, Utrecht, The Netherlands) at a rate of $15 \mathrm{~mL} / \mathrm{kg} / \mathrm{h}$ and maintenance of anesthesia. The right femoral artery was cannulated for drawing blood samples and the right femoral vein for drug administration.

The left kidney was exposed, decapsulated, and immobilized in a lucite kidney cup (K. Effenberger, Pfaffingen, Germany) via $\sim 4 \mathrm{~cm}$ incision in the left flank in each animal. Renal vessels were carefully separated under preservation of nerves and the adrenal gland. A perivascular ultrasonic transient time flow probe was placed around the left renal artery (type 0.7 RB Transonic Systems Inc., Ithaca, NY, USA) and connected to a flow meter (T206, Transonic Systems Inc., Ithaca, NY, USA) to continuously measure renal blood flow (RBF). An estimation of the renal vascular resistance (RVR) was made as: RVR (dynes.sec. $\mathrm{cm}^{-5}$ ) = $(\mathrm{MAP} / \mathrm{RBF})$. The left ureter was isolated, ligated, and cannulated with a polyethylene catheter for urine collection. 
After the surgical preparation, one optical fiber was placed $1 \mathrm{~mm}$ above the decapsulated kidney and another optical fiber was placed $1 \mathrm{~mm}$ above the renal vein to measure renal microvascular and venous oxygenation using phosphorimetry (explained in more detail below). A small piece of aluminum foil was placed on the dorsal side of the renal vein to prevent contribution of the underlying tissues to the phosphorescence signal in the venous $\mathrm{pO}_{2}$ measurements. Oxyphor G2, a two-layer glutamate dendrimer of tetra-(4-carboxy-phenyl) benzoporphyrin (Oxygen Enterprises Ltd., Philadelphia, PA, USA) was subsequently infused (i.e., $6 \mathrm{mg} / \mathrm{kg}$ i.v. over $5 \mathrm{~min}$ ), followed by $30 \mathrm{~min}$ of stabilization time. The surgical field was covered with a humidified gauze compress throughout the entire experiment to prevent drying of the exposed tissues.

\section{Experimental Protocol}

The rats were divided into three groups ( $n=6$ /group): (1) a sham-operated control group; (2) a group subjected to renal ischemia for $60 \mathrm{~min}$, by high aortic occlusion with a custom-made vascular occluder placed on the abdominal aorta just above mesenteric artery, followed by $2 \mathrm{~h}$ of reperfusion (I/R); and (3) a group subjected to I/R and treated with ascorbic acid: at $15 \mathrm{~min}$ before ischemia, an i.v. bolus ( $100 \mathrm{mg} / \mathrm{kg}$ dissolved in $1 \mathrm{~mL} 0.09 \%$ saline) was administered for $5 \mathrm{~min}$, followed by continuous i.v. infusion (50 mg/kg/h dissolved in $0.5 \mathrm{~mL} 0.09 \%$ saline) for $2 \mathrm{~h}$ during reperfusion $(\mathrm{I} / \mathrm{R}+\mathrm{AA})$.

\section{Blood variables}

Arterial blood samples $(0.5 \mathrm{~mL})$ were taken from the carotid artery at three time points: (1) before aortic occlusion, (baseline, BL); (2) 15 min. after reperfusion (initial reperfusion phase, R15); and (3) $120 \mathrm{~min}$. after reperfusion (late reperfusion phase, R120). The blood samples were replaced by the same volume of HES130/0.4 (Voluven, 6\% HES 130/0.4; Fresenius Kabi Nederland, Schelle, Belgium). The samples were used for determination of blood gas values (ABL505 blood gas analyzer; Radiometer, Copenhagen, Denmark), as well as for determination of the hemoglobin concentration, hemoglobin oxygen saturation (OSM 3; Radiometer).

\section{Renal microvascular and venous oxygenation}

Microvascular oxygen tension in the renal cortex $\left(\mathrm{C}_{\mu} \mathrm{PO}_{2}\right)$, outer medulla $\left(\mathrm{M \mu PO}_{2}\right)$, and renal venous oxygen tension $\left(\mathrm{P}_{\mathrm{rv}} \mathrm{O}_{2}\right)$ were measured by oxygen-dependent quenching of phosphorescence lifetimes of the systemically infused albumin-targeted (and therefore circulation-confined) phosphorescent dye Oxyphor G2 ${ }^{[28]}$. Oxyphor G2 has two excitation peaks $\left(\lambda_{\text {excitation1 } 1} 440 \mathrm{~nm}, \lambda_{\text {excitation } 2}=632 \mathrm{~nm}\right)$ and one emission peak $\left(\lambda_{\text {emission }}=800 \mathrm{~nm}\right)$. These optical properties allow (near) simultaneous lifetime measurements in microcirculation of the kidney cortex and the outer medulla due to different optical penetration depths of the excitation light ${ }^{[28]}$. For the measurement of renal venous $\mathrm{PO}_{2}$ $\left(\mathrm{P}_{\mathrm{rv}} \mathrm{O}_{2}\right)$, a mono-wavelength phosphorimeter was used ${ }^{[29]}$. Oxygen measurements based on phosphorescence lifetime techniques rely on the principle that phosphorescence can be quenched by energy transfer to oxygen resulting in shortening of the phosphorescence lifetime. A linear relationship between reciprocal phosphorescence lifetime and oxygen tension (i.e., the Stern-Volmer relation) allows quantitative measurement of $\mathrm{PO}_{2}^{[30]}$.

\section{Renal oxygen delivery and consumption}

Arterial oxygen content (AOC) was calculated by (1.31 $\times$ hemoglobin $\left.\times \mathrm{S}_{\mathrm{a}} \mathrm{O}_{2}\right)+\left(0.003 \times \mathrm{P}_{\mathrm{a}} \mathrm{O}_{2}\right)$, where $\mathrm{S}_{\mathrm{a}} \mathrm{O}_{2}$ is arterial oxygen saturation and $\mathrm{P}_{\mathrm{a}} \mathrm{O}_{2}$ is arterial partial pressure of oxygen. Renal venous oxygen content (RVOC) was calculated as $\left(1.31 \times\right.$ hemoglobin $\left.\times \mathrm{S}_{\mathrm{rV}} \mathrm{O}_{2}\right)+(0.003 \times$ $\mathrm{P}_{\mathrm{rv}} \mathrm{O}_{2}$ ), where $\mathrm{S}_{\mathrm{rv}} \mathrm{O}_{2}$ is venous oxygen saturation and $\mathrm{P}_{\mathrm{rv}} \mathrm{O}_{2}$ is renal vein partial pressure of oxygen (measured using phosphorimetry). Renal oxygen delivery was calculated as $\mathrm{DO}_{2}(\mathrm{~mL} / \mathrm{min})=\mathrm{RBF} \times$ AOC. Renal oxygen consumption was calculated as $\mathrm{VO}_{2}(\mathrm{~mL} / \mathrm{min})=\mathrm{RBF} \times(\mathrm{AOC}-\mathrm{RVOC})$.

\section{Renal function}

Creatinine was determined in plasma at the end of the experiment. We aimed to collect urine samples from the left ureter, but due to the severity of our model, no urine was produced after I/R.

\section{Renal tissue oxidative stress}

Renal tissue malondialdehyde (MDA) levels were determined to assess lipid peroxidation as a measure of renal oxidative stress. At the end of the experiment, the left kidney was cut in two, and one-half was immediately frozen in liquid nitrogen and stored at $-80^{\circ} \mathrm{C}$. Frozen kidneys were homogenized in cold $5 \mathrm{mM}$ sodium phosphate buffer. The homogenates were centrifuged at $12,000 \mathrm{~g}$ for $15 \mathrm{~min}$ at $4^{\circ} \mathrm{C}$ and supernatants were used for MDA determination. The amount of MDA was quantified using a Quattro Premier XE tandem mass spectrometer (MS/MS) from Waters (Milford, MA, USA) with aAcquity sample manager and a Acquity binary solvent manager. MDA and MDA-d2 were separated on a Supelco LC-18DB column $(250 \mathrm{~mm}$-length $\times 4.6 \mathrm{~mm}$-diameter, with $5 \mu \mathrm{m}$ particles), using a isocratic run from $50 \%$ acetonitrile, $50 \%$ water and $0.2 \%$ Acetic acid; the flow rate was $1 \mathrm{~mL} / \mathrm{min}$ with a total run time of $10 \mathrm{~min}$. Both compounds were detected and quantified by MRM acquisition in positive electrospray ionization mode, using the transitions $\mathrm{m} / \mathrm{z} 235$ $>159$ for MDA, and $237>161$ for MDA-d2.

\section{Immunohistochemistry}

At the end of the experiment, the other half of the left kidney was fixed in 4\% formalin and embedded in paraffin. Kidney sections $(4 \mu \mathrm{m})$ were deparaffinized with xylene and rehydrated with decreasing percentages of ethanol 
and finally, with water. Antigen retrieval was accomplished by microwaving slides in citrate buffer ( $\mathrm{pH}$ 6.0) (Thermo Scientific, AP-9003-500) for $10 \mathrm{~min}$. Slides were left to cool for $20 \mathrm{~min}$ at room temperature and then rinsed with distilled water. Surroundings of the sections were marked with a PAP pen. The endogenous peroxidase activity was blocked with $3 \% \mathrm{H}_{2} \mathrm{O}_{2}$ for $15 \mathrm{~min}$ at room temperature and later rinsed with distilled water and PBS. Blocking reagent (Lab Vision, TA-125-UB) was applied to each slide followed by 5 min incubation at room temperature in a humid chamber. Kidney sections were incubated overnight at $4^{\circ} \mathrm{C}$ with rabbit polyclonal iNOS antibody (iNOS Ab1, Rabbit PAb, RB-1605-P, NeoMarkers Fremont, CA) and IL-6 (Abcam, 6672), and incubated for $1 \mathrm{~h}$ at room temperature with anti-myeloperoxidase (MPO) antibodies (Myeloperoxidase Ab-1, RB-373-A, NeoMarkers Fremont, CA), Lipocalin 2 antibody (NGAL, neutrophil gelatinaseassociated lipocalin) (abcam 41105), polyclonal antibody to rat L-FABP, liver-fatty acid binding protein (HycultBiotect HP8010). Antibodies were diluted in a large volume of UltrAb Diluent (Thermo Scientific, TA-125-UD). The sections were washed in PBS three times for 5 min each time and then incubated for $30 \mathrm{~min}$ at room temperature with biotinylated goat anti-rabbit antibodies (LabVision, TP-125-BN). After slides were washed in PBS, the streptavidin peroxidase label reagent (LabVision, TS-125HR) was applied for $30 \mathrm{~min}$ at room temperature in a humid chamber. The colored product was developed by incubation with 3-amino-9-ethylcarbazole (AEC). The slides were counterstained with Mayer's hematoxylin (LabVision, TA-125-MH) and mounted in vision mount (LabVision, TA-060-UG) after being washed in distilled water. Both the intensity and distribution of iNOS, IL-6, L-FABP, NGAL staining were scored in 10 different areas for each samples under light microscope at magnification X400 in the kidney slices. For each sample, a histological score (HSCORE) value was derived by summing the percentages of cells that stained at each intensity multiplied by the weighted intensity of the staining [HSCORE $=\mathrm{SPi}(i+1)$, where $i$ is the intensity score and $\mathrm{P} i$ is the corresponding percentage of the cells]. We evaluated MPO reaction in the glomerulus from 250 selected glomeruli and in 250 selected peritubular areas under a light microscope at a magnification X400. We scored 1 if leukocytes could be seen in the glomerulus or peritubular areas and 0 if not ${ }^{[9,31]}$.

\section{Statistical Analysis}

Data analysis and presentation were performed using GraphPad Prism (GraphPad Software, San Diego, CA, USA). Values are reported as the mean \pm SD. Two-way analysis of variance (ANOVA) for repeated measurements with a Bonferroni post hoc test were used for comparative analysis between different time points of the groups. The repeated-measures ANOVA (one-way with Bonferroni post hoc test) was used for comparative analysis between groups. $P$-value of $<0.05$ was considered statistically significant.

\section{RESULTS}

\section{Systemic and renal hemodynamic}

Systemic and renal hemodynamic parameters are reported in Table 1. High aortic clamping and release resulted in a significant fall in blood pressure and RBF at R15 and R120 ( $P<0.05 v s$. time control), and a significant increase in RVR at R120 ( $P<0.05$ vs. time control). Although AA treatment was associated with a non-significant trend for improved MAP and RBF, this only resulted in a significant improvement for RVR at R120 $(P<0.05 v$ s. I/ R + AA group). MAP and RBF remained significantly decreased in the I/R + AA group compared to the time control group.

\section{Renal oxygenation}

Renal oxygenation parameters are reported in Figures 1 and 2. Briefly, renal $\mathrm{DO}_{2 \text { ren }}, \mathrm{VO}_{2 \text { ren }}, \mathrm{CmPO}_{2}$, and $\mathrm{MmPO}_{2}$

\begin{tabular}{|c|c|c|c|}
\hline & $B L$ & R15 & R120 \\
\hline \multicolumn{4}{|l|}{ MAP [mmHg] } \\
\hline Time control & $87 \pm 7$ & $88 \pm 21$ & $69 \pm 7$ \\
\hline $\mathrm{I} / \mathrm{R}$ & $84 \pm 3$ & $44 \pm 8^{*}$ & $34 \pm 7^{*}$ \\
\hline $\mathrm{I} / \mathrm{R}+\mathrm{AA}$ & $93 \pm 14$ & $48 \pm 14^{*}$ & $46 \pm 14^{*}$ \\
\hline \multicolumn{4}{|l|}{ RBF $[\mathrm{mL} / \mathrm{min}]$} \\
\hline Time control & $4.1 \pm 1.0$ & $3.4 \pm 1.2$ & $3.8 \pm 0.9$ \\
\hline $\mathrm{I} / \mathrm{R}$ & $4.6 \pm 1.0$ & $1.6 \pm 0.2^{*}$ & $0.6 \pm 0.2^{*}$ \\
\hline $\mathrm{I} / \mathrm{R}+\mathrm{AA}$ & $4.6 \pm 0.8$ & $1.4 \pm 0.4^{*}$ & $1.2 \pm 0.4^{*}$ \\
\hline \multicolumn{4}{|c|}{ RVR [dyn.s.cm - 5] } \\
\hline Time control & $23 \pm 7$ & $28 \pm 9$ & $19 \pm 4$ \\
\hline $\mathrm{I} / \mathrm{R}$ & $19 \pm 5$ & $28 \pm 4$ & $54 \pm 10^{*}$ \\
\hline $\mathrm{I} / \mathrm{R}+\mathrm{AA}$ & $21 \pm 4$ & $35 \pm 10$ & $34 \pm 6^{*},+$ \\
\hline
\end{tabular}

I/R: ischemia/reperfusion; AA: ascorbic acid. Values are represented as a mean $\pm \mathrm{SD},{ }^{*} P<0.05 v s$. time control; $+P<0.05 v s$. I/R. 
decreased slightly after releasing of the clamp at R15 and R120 ( $P<0.05$ vs. time control, respectively) ( Figure 1). AA treatment improved $\mathrm{CmPO}_{2}$ in I/R group at R15 and $\mathrm{R} 120$ ( $P<0.05 v$ s. $\mathrm{I} / \mathrm{R}$, respectively), whereas MmPO2 was improved only at R120 $(P<0.05 v s$. I/R) (Figures $1 \mathrm{C}$ and 1D). Additionally, while $\mathrm{CmPO} 2$ decreased by $\sim 23 \%$ in the control group, by $\sim 75 \%$ in the $\mathrm{I} / \mathrm{R}$ group $(P<0.05 v$ s. time control), and by $\sim 51 \%$ in the $\mathrm{I} / \mathrm{R}+\mathrm{AA}$ group $(P<0.05$ $v s$. time control), which also indicated an improvement in
$\mathrm{I} / \mathrm{R}$ group treated with $\mathrm{AA}$ in comparison to $\mathrm{I} / \mathrm{R}$ group $(P<0.05)$ as a percentage from BL to R120. MmPO2 decreased by $\sim 19 \%$ in the control group, by $\sim 80 \%$ in the I/R group $(P<0.05 v$ s. time control $)$ and $\sim 59 \%$ in the I/ $\mathrm{R}$ + AA group $(P<0.05 v$ s. time control $)$ resulted significant improvement in the $\mathrm{I} / \mathrm{R}$ group received AA compared with the I/R group $(P<0.05)$ (Figures $2 \mathrm{C}, 2 \mathrm{D})$. Overall, the data suggest that AA treatment can, at least, partly improve the I/R-induced hypo-oxygenation of the kidney.

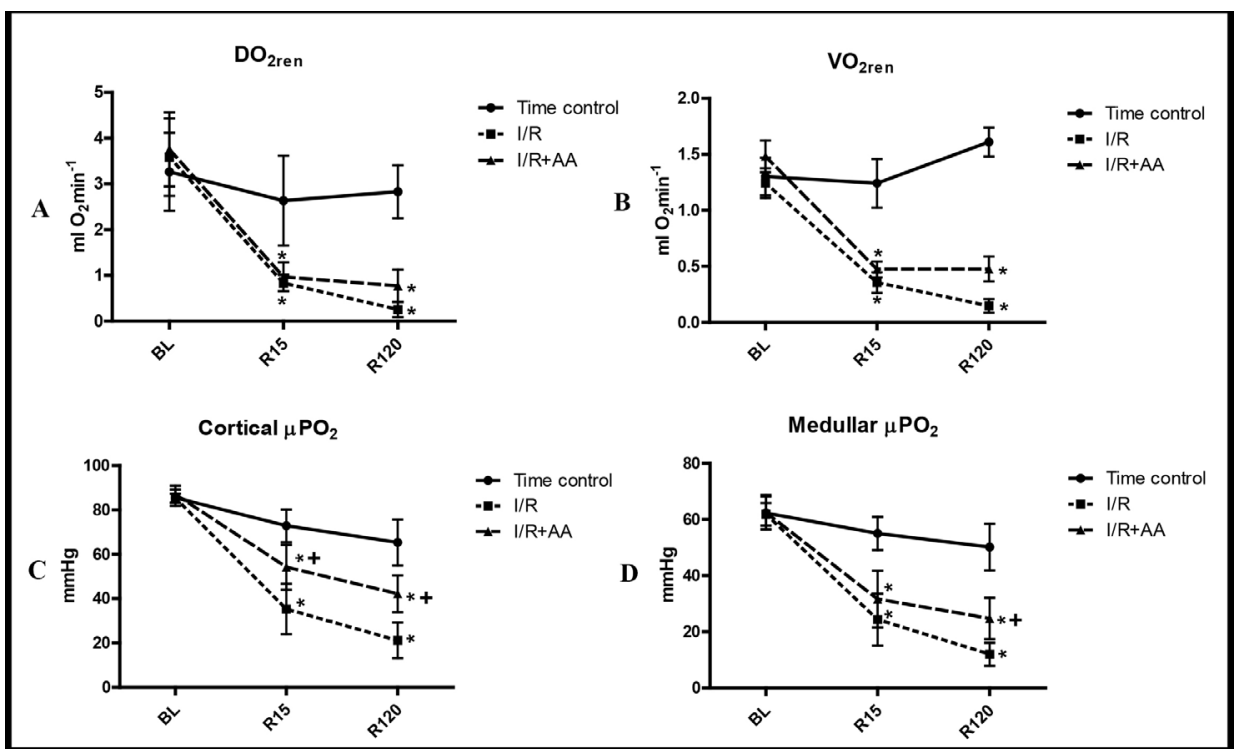

Figure 1: Renal oxygen delivery $\left(\mathrm{DO}_{2 \text { ren }}\right)($ Panel $\mathrm{A})$, renal oxygen consumption $\left(\mathrm{VO}_{2 \text { ren }}\right)($ Panel $\mathrm{B})$, cortical microvascular oxygen pressure $\left(\right.$ cortical $\left.\mathrm{mPO}_{2}\right)\left(\mathrm{Panel}_{\mathrm{C}}\right)$ and medullar microvascular oxygen pressure (medullar $\left.\mathrm{mPO}_{2}\right)($ Panel $\mathrm{D})$ during experiments. I/R: ischemia/reperfusion; $\mathrm{AA}$ : ascorbic acid. Values are presented as a mean \pm SD. ${ }^{*} P<0.05$ vs. time control, $+P<0.01$ vs. I/R.

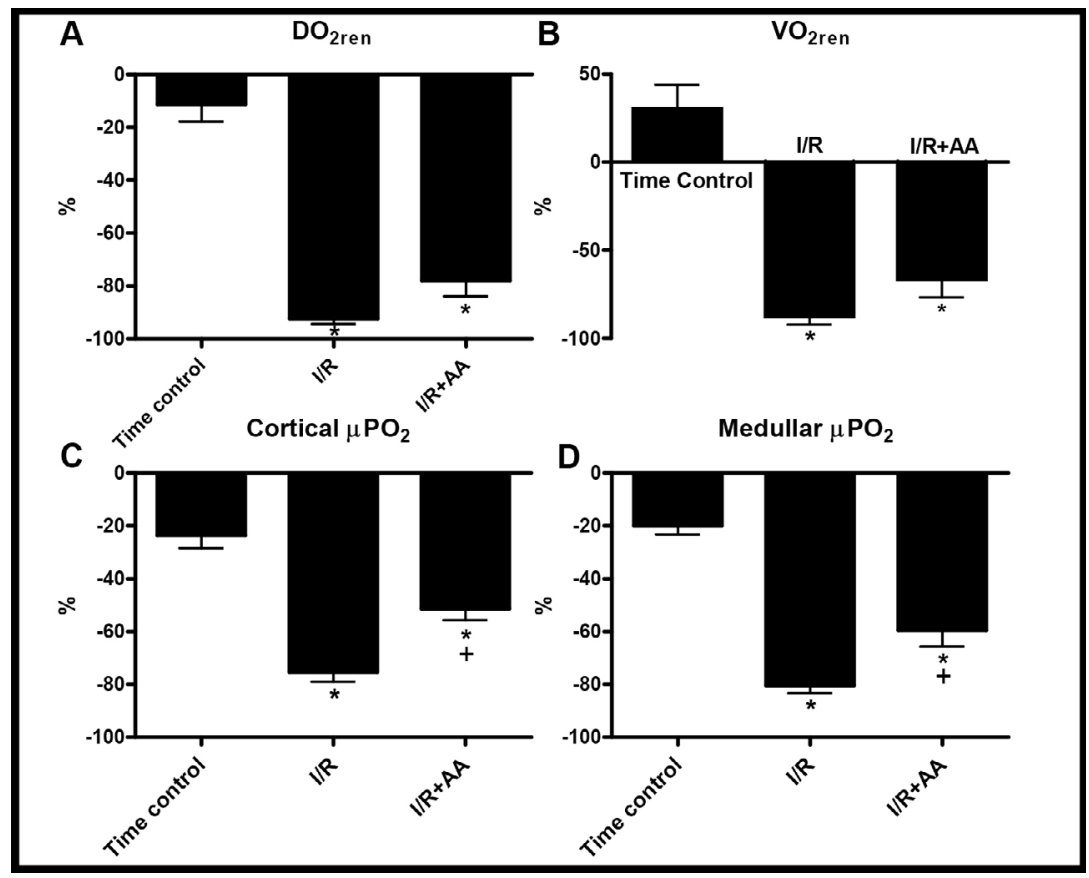

Figure 2: Changing values of renal oxygen delivery $\left(\mathrm{DO}_{2 \text { ren }}\right)($ Panel $\mathrm{A})$, renal oxygen consumption $\left(\mathrm{VO}_{2 \text { ren }}\right)($ Panel $\mathrm{B})$, cortical microvascular oxygen pressure $\left(\right.$ cortical mPO $\left.\mathrm{m}_{2}\right)$ $($ Panel $\mathrm{C})$ and medullar microvascular oxygen pressure (medullar $\left.\mathrm{mPO}_{2}\right)($ Panel $\mathrm{D})$ from $\mathrm{BL}$ to $\mathrm{R} 120$ between groups. I/R: ischemia/reperfusion; AA: ascorbic acid. Values are presented as a mean $\pm \mathrm{SD}$. ${ }^{*} P<0.05$ vs. time control, $+P<0.01 \mathrm{vs.} \mathrm{I/R}$. 


\section{Plasma lactate levels}

In accordance with the I/R-induced hypo-oxygenation of the kidney, untreated $\mathrm{I} / \mathrm{R}$ was associated with large increases in plasma lactate levels, from $2 \pm 1 \mathrm{mmol} / \mathrm{L}$ in the control group to $13 \pm 4 \mathrm{mmol} / \mathrm{L}$ in the $\mathrm{I} / \mathrm{R}$ group $(P<0.05)$. The rise in lactate levels was significantly reduced to $7 \pm 4 \mathrm{mmol} / \mathrm{L}$ by $\mathrm{AA}$ administration with respect to I/R group $(P<0.05)$ (Figure 3).

\section{Renal function parameters}

We aimed to collect urine samples from the left ureter for analysis of urine volume, creatinine concentration, and sodium concentration at the end of the protocol, but due to the severity of our model (severe fall in MAP, $\mathrm{RBF}$, and renal oxygenation and a significant rise in RVR), no urine was produced after I/R. AA treatment was unable to restore this blocked urine production. Plasma creatinine levels were higher after the I/ $\mathrm{R}$ insult at the end of the experiment, suggesting decreased kidney function. However, AA treatment was also unable to attenuate the $\mathrm{I} / \mathrm{R}$-induced creatinine increase (Figure 4).

\section{Immunohistochemistry}

Next, we examined whether the decreased oxidative stress by AA treatment resulted in attenuation of I/R-induced inflammation and kidney damage (Figure 5). In comparison to the control group, I/R led to a significant rise in all immunohistochemistry parameters: iNOS, IL-6, neutrophil gelatinase-associated lipocalin (NGAL), fatty acid binding protein (FABP), and myeloperoxidase (MPO) expression in the glomeruli (gMPO) and peritubular areas (ptMPO) $(P<0.05)$. Ascorbic acid treatment was able to prevent the $\mathrm{I} / \mathrm{R}$-induced rise in all of these parameters $(P<0.05)$ with respect to the $\mathrm{I} / \mathrm{R}$ group.

\section{Renal tissue oxidative stress}

We first assessed whether oxidative stress developed and to what extent AA treatment was effective in reducing oxidative stress in our model. Renal tissue MDA levels were determined to assess lipid peroxidation as a measure of renal oxidative stress. I/ $\mathrm{R}$ led to a significant increase in MDA levels from $4.9 \pm 1.3 \mathrm{mmol} / \mathrm{g}$ in the time control group to $7.6 \pm 1.3 \mathrm{mmol} / \mathrm{g}$ in the $\mathrm{I} / \mathrm{R}$ group $(P<0.05)$, which was decreased to control levels $(5.3 \pm 0.9 \mathrm{mmol} / \mathrm{g})$ by ascorbic acid administration $(P<0.05)$ (Figure 6$)$.

\section{DISCUSSION}

In the present study, we investigated whether the ascorbic acid (vitamin C) administration would be able to reduce $\mathrm{I} / \mathrm{R}$-related injury to the kidneys in terms of renal microvascular hypoxia, oxidative stress, inflammation, and finally, renal failure. The severe I/ $\mathrm{R}$ intervention resulted in a fall in systemic and renal hemodynamics and oxygenation

\section{Lactate}

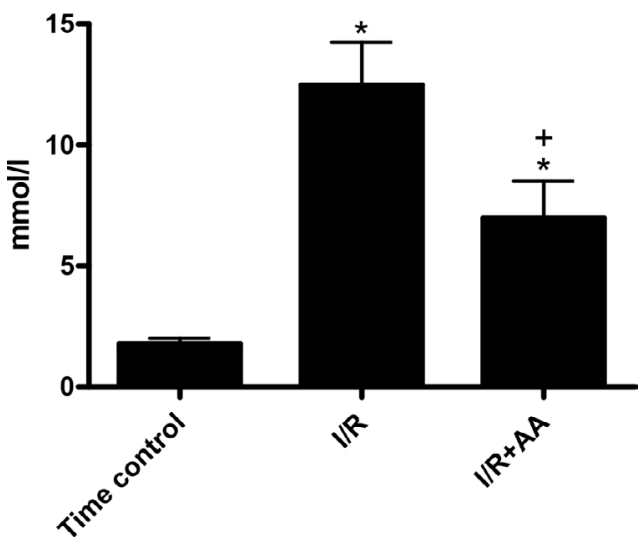

Figure 3: Plasma lactate levels at the end of the protocol (R120). I/R: ischemia/ reperfusion; AA: ascorbic acid. Values are presented as a mean $\pm S D$, ${ }^{*} P<0.05$ vs. time control and $+P<0.05$ vs. I/R.

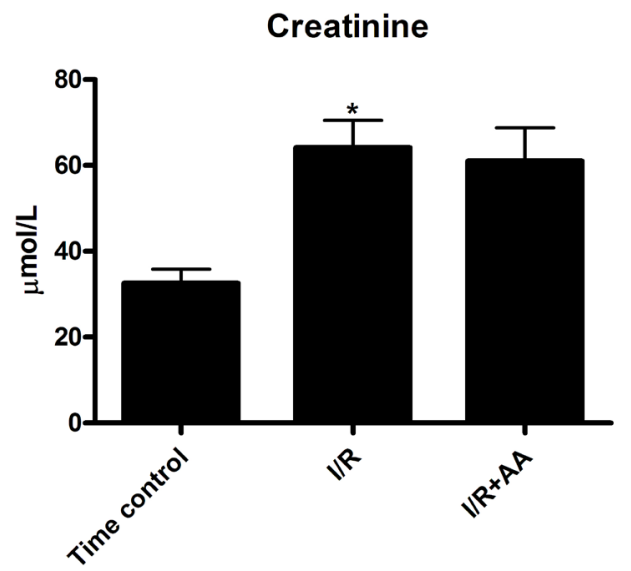

Figure 4: Plasma creatinine levels at the end of the protocol (R120). I/R: ischemia/ reperfusion; AA: ascorbic acid. Values are presented as a mean $\pm S D,{ }^{*} P<0.05$ vs. time control.

parameters, and significantly increased the expression of biomarkers of kidney injury and inflammation. This was also associated with complete anuria and elevated plasma creatinine and lactate levels. However, treatment of ascorbic acid resulted in improvement in the renal cortical and medulla oxygenation and partially enhanced in renal oxygen delivery and consumption as a result of a decreased amount of oxygen using for the reactive oxygen species production. We demonstrated that ascorbic acid administration was able to completely prevent the burst in oxidative stress, inflammation, and renal injury. However, the AA was unable to improve hemodynamics or urine function in this severe model of AKI.

Many regulatory factors contribute to the homeostasis of oxygen supply and consumption of the kidney ${ }^{[18]}$, including the systemic and microvascular hemodynamic circumstances. To demonstrate complexity of the renal 


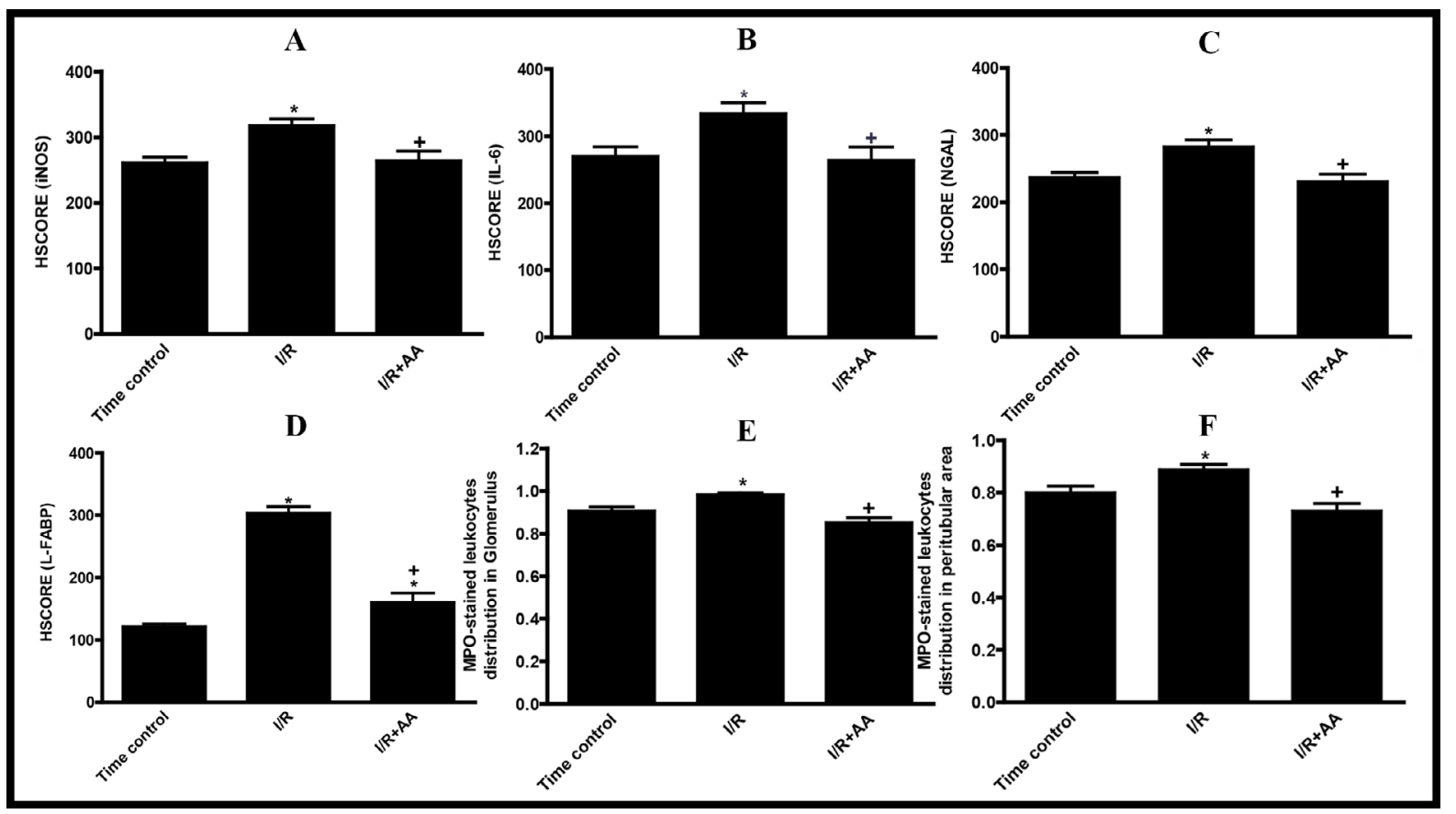

Figure 5: The immunohistochemistry results for expression of inducible nitric oxide (iNOS) (Panel A), interleukin 6 (IL-6) (Panel B), neutrophil gelatinase-associated lipocalin (NGAL) (Panel C), fatty acid binding protein (FABP) (Panel D), and myeloperoxidase (MPO) in the glomeruli (gMPO) (Panel E) and peritubular areas (ptMPO) (Panel F). I/R: ischemia/reperfusion; AA: ascorbic acid. Values are represented as mean $\pm \mathrm{SD}$, ${ }^{*} P<0.05$ vs. time control; $+P<0.05 \mathrm{vs}$. I/R.

oxygenation and perfusion, $\mathrm{O}^{\prime}$ Connor et al., reported that reductions in renal blood flow (RBF), instigated by direct injection of vasopressin into the renal artery, led to a selective reduction in medullar perfusion and $\mathrm{PO}_{2}$, without affecting cortical perfusion or tissue oxygenation. On the other hand, stimulation of the renal nerves caused renal cortical ischemia associated with reductions in renal flow, cortical perfusion, and cortical $\mathrm{PO}_{2}{ }^{[32]}$. In the present study, we have documented that macrohemodynamic changes such as MAP and RBF are associated with alteration of the renal microvascular and oxygenation properties as reflected by a change in the renal vascular resistance. Recently, a few studies suggested that oxygen shunting may also contribute to renal microcirculatory disturbance and oxygen heterogeneity ${ }^{[10,33]}$, which shunting possibly being an adaptation to prevent overproduction of the reactive oxygen species $(\mathrm{ROS})^{[6]}$. Legrand et al. suggested that the homeostasis of oxygen supply and utilization is severely impaired during ischemia/reperfusion in kidney ${ }^{[18]}$. In this study, we found that I/R-induced impairment of renal cortical and medullar microcirculatory oxygenation correlates with the deterioration of $\mathrm{DO}_{2 \text { ren }}$ and $\mathrm{VO}_{2 \text { ren }}$. Legrand et al. also documented that microcirculatory oxygen disturbance and tissue hypoxia occurred during the first hours of the reperfusion, and correlated with the reduction of $\mathrm{DO}_{2 \text { ren }}$ and $\mathrm{VO}_{2 \text { ren }}$ in both renal cortex and medulla. As an additional correlate of oxygen homeostasis disturbance, we also observed elevated plasma lactate levels after I/R. These microcirculatory disturbances were previously

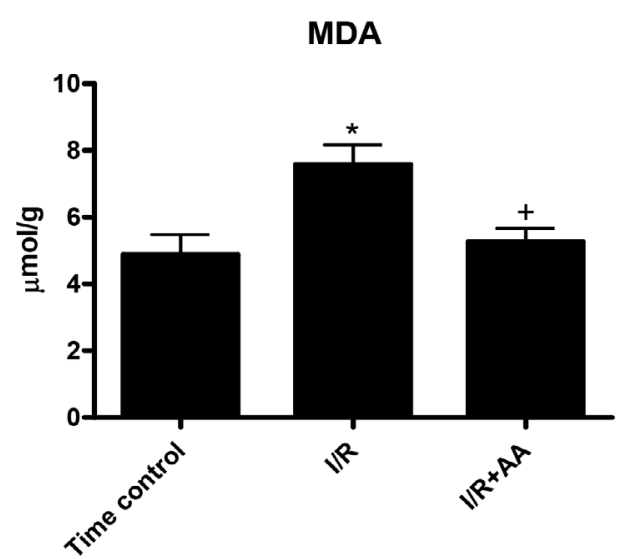

Figure 6: Renal tissue malondialdehyde (MDA) levels at the end of the protocol (R120). I/R: ischemia/reperfusion; AA: ascorbic acid. Values are presented as a mean $\pm \mathrm{SD},{ }^{*} P<0.05 \mathrm{vs}$. Time control and ${ }^{+} P<0.05 \mathrm{vs}$. I/R.

shown to be partly mediated through upregulation of iNOS and downregulation of eNOS ${ }^{[9,10]}$. In fact, nitric oxide (NO) is of prime importance because of its central regulatory role in intrarenal microcirculation ${ }^{[34]}$ and renal oxygen consumption regulation ${ }^{[35,36]}$. In the present study, we also demonstrated that severe hemodynamic alterations are associated with elevating iNOS activity.

In the present study, we used $\mathrm{AA}$, which is one of the water-soluble vitamins and is well known as a strong antioxidant agent reacting with and scavenging superoxide, hydroxyl radicals, and singlet oxygen ${ }^{[37]}$. Tyml et al. have also demonstrated that an intravenous bolus of ascorbate could 
protect against maldistribution of microvascular blood flow and reversed microcirculatory dysfunction in sepsis ${ }^{[38]}$. In present study, we demonstrated that intravenous AA administration could improve the level of lactate in plasma as a result of its protective effect on the microvascular circulation and oxygenation.

It has been documented that $\mathrm{AA}$ also reduces peroxynitrite formation, which is a strong oxidant molecule and is mainly generated when overexpressed NO reacts with singlet oxygen. Recently, Wang et al. ${ }^{[3]]}$ have also demonstrated that levels of the nuclear factor-kB (NF-kB) and iNOS mRNA were elevated in ischemia-reperfused kidney, resulting in an increased peroxynitrite formation and lipid peroxidation. Additionally, another study showed that peroxynitrite can lead to inhibition of mitochondrial respiratory chain activity and membrane $\mathrm{Na} / \mathrm{K}$-ATPase activity, and activation of apoptotic enzymes ${ }^{[40]}$. Thus, it might be hypothesized that peroxynitrite is able to impair the renal microcirculatory oxygenation and utilization by inhibition of the $\mathrm{Na} / \mathrm{K}$ ATPase and mitochondrial respiratory chain enzymes. Indeed, under physiological conditions, approximately $80 \%$ of oxygen is used to drive the $\mathrm{Na} / \mathrm{K}$-ATPase in kidney ${ }^{[41]}$. In the present study, we concluded that although ascorbic acid could restore renal cortical and medullar oxygenation, and marginally improved in the renal oxygen delivery and consumption. These oxygenation results also indicated that $\mathrm{AA}$ was able to prevent peroxynitrite formation and was effective in the oxygen utilization by decreasing iNOS levels and peroxynitrite formation in this study. However, this cortico-medullary microcirculatory effect of AA can be explained through inhibition of the $\mathrm{A}-\mathrm{V}$ shunting process due to reduction in oxidative stress and supplying sufficient oxygen in the renal microvasculature by protecting the endothelium and reduction of the RVR by local vessel vasodilatation even though unimproved RBF and arterial pressure because of consistent hypotension.

Several studies have demonstrated that I/R in the kidney is associated with lipid peroxidation, which is an autocatalytic mechanism leading to oxidative destruction of cellular membranes ${ }^{[42,43]}$. In accordance, renal MDA level was also significantly increased in our model indicating the presence of enhanced lipid peroxidation due to $\mathrm{I} / \mathrm{R}$ injury ${ }^{[4]}$. In the present study, we demonstrated that the ascorbic acid was able to completely prevent the I/R-induced increase in tissue MDA levels. However, kidney function was not completely restored indicating that oxidative stress is not the only causal factor for the development of AKI. Furthermore, we showed that ascorbic acid also attenuates leukocyte activation and leukocyte adhesion to microvascular endothelium by assessment in renal tissue levels of MPO and IL-6. Previous studies demonstrated that inflammatory molecules are expressed in a few minutes of $\mathrm{I} / \mathrm{R}$ injury, including platelet-activating factor (PAF) and $\mathrm{P}$-selectin, which is associated with activation of the complement system, release of leukocyte chemotactic factors ${ }^{[4]}$, and upregulation of adhesion molecules on the cell surface, leading to adherence of circulating leukocytes to the vessel walls and their diapedesis into the tissues ${ }^{[4]}$. Our results are in support of the findings by Lloberas et al. ${ }^{[47]}$, showing AA-induced attenuation of PAF activation and leukocyte recruitment in kidney.

This study has several limitations. First of all, the 2-h measurement period post-ischemia might be considered too short for assessment of the actual impact of $\mathrm{I} / \mathrm{R}$ injury on kidney. However, the aim of this study was first to focus on renal hemodynamic and oxygenation changes in early stage of I/R. Furthermore, our results clearly demonstrate the initiation of renal dysfunction within this acute phase. A further important limitation of the study is that the procedure we chose resulted in acute hypotension and shock as indicated by the fall in MAP and rise of lactate. In fact, simply administrating AA was not able to restore macrohemodynamic parameters and this could also underlie our finding of the inability of only AA administration to restore kidney. Co-adminstering fluids with AA and targeting the blood pressure could have modulated this effect and maybe have had a more beneficial effect on renal function. However we did not want to mix these two therapies in the present study. The severity of the $\mathrm{I} / \mathrm{R}$ injury led to anuria, that is why we could not determine creatinine clearance and sodium reabsorption and instead, focused our attention on biomarkers of renal injury such as NGAL and FABP.

\section{CONCLUSIONS}

In this study, we have demonstrated that acute ischemia/ reperfusion cause an impairment of kidney cortical and medullar oxygenation. These microcirculatory alterations are associated with decreases in renal oxygen supply and oxygen consumption, and increases in renal vascular resistance. At the same time, these changes were also correlated with systemic hypotension, tissue damage, inflammatory activation, and oxidative stress. Finally, assuming oxidative stress and microcirculatory disturbance to be an important contributor to I/R-induced AKI, we examined whether the ascorbic acid can prevent oxidative stress and therefore, I/R-induced microcirculatory disturbance. We observed that the ascorbic acid was able to completely prevent the rise in oxidative stress, and partly improved renal microcirculatory oxygenation with reductions in kidney inflammation and tissue damage. However, the mitigation of oxidative stress was unable to restore kidney function in this rat model of severe ischemia/ reperfusion. To conclude, we suggested that ascorbic acid 
might be used for prophylactic and therapeutic agent in order to prevent I/R-induced oxidative stress and renal oxygenation disturbance along with the fluid replacement and vasoconstrictor therapy against hypotension.

\section{Acknowledgment}

This study was carried out as a part of project supported by the Dutch Kidney Foundation (grant C 09.2290), Bussum, The Netherlands.

\section{Conflicts of Interest}

Prof. Can Ince has received a grand and consultancies from Baxter, Fresenius Kabi, and B Braun. For remaining authors non conflicts of interest were declared.

\section{REFERENCES}

1. Rabb H, Wang Z, NemotoT, Hotchkiss J, Yokota N, Soleimani M. Acute renal failure leads to dysregulation of lung salt and water channels. Kidney Int 2003; 63: 600-6.

2. Evans RG, Ince C, Joles JA, Smith DW, May CN, O'Connor PM, et al. Haemodynamic influences on kidney oxygenation: clinical implications of integrative physiology. Clin Exp Pharmacol Physiol 2013; 40: 106-22.

3. Le Dorze M, Legrand M, Payen D, Ince C. The role of the microcirculation in acute kidney injury. Curr Opin Crit Care 2009; 15: 503-8.

4. Singh P, Ricksten SE, Bragadottir G, Redfors B, Nordquist L. Renal oxygenation and haemodynamics in acute kidney injury and chronic kidney disease. Clin Exp Pharmacol Physiol 2013; 40: 138-47.

5. Evans RG, Eppel GA, Michaels S, Burke SL, Nematbakhsh M, Head GA, et al. Multiple mechanisms act to maintain kidney oxygenation during renal ischemia in anesthetized rabbits. Am J Physiol Renal Physiol 2010; 298: F1235-43.

6. Evans RG, Gardiner BS, Smith DW, O'Connor PM. Intrarenal oxygenation: Unique challenges and the biophysical basis of homeostasis. Am J Physiol Renal Physiol 2008; 295: F1259-70.

7. Warner L, Gomez SI, Bolterman R, Haas JA, Bentley MD, Lerman LO, et al. Regional decreases in renal oxygenation during graded acute renal arterial stenosis: a case for renal ischemia. Am J Physiol Regul Integr Comp Physiol 2009; 296: R67-71.

8. Abdelkader A, Ho J, Ow CP, Eppel GA, Rajapakse NW, Schlaich MP, et al. Renal oxygenation in acute renal ischemia-reperfusion injury. Am J Physiol Renal Physiol 2014; 306: F1026-38.

9. Legrand M, Almac E, Mik EG, Johannes T, Kandil A, Bezemer R, et al. L-NIL prevents renal microvascular hypoxia and increase of renal oxygen consumption after ischemia-reperfusion in rats. Am J Physiol Renal Physiol 2009; 296: F1109-17.

10. Legrand M, Kandil A, Payen D, Ince C. Effects of sepiapterin infusion on renal oxygenation and early acute renal injury after suprarenal aortic clamping in rats. J Cardiovasc Pharmacol 2011; 58: 192-8.

11. Parrino PE, Laubach VE, Gaughen JR, Shockey KS, Wattsman TA, King $\mathrm{RC}$, et al. Inhibition of inducible nitric oxide synthase after myocardial ischemia increases coronary flow. Ann Thorac Surg 1998; 66:733.

12. Koksel O, Ozdulger A, Aytacoglu B, Polat A, Sucu N, Yildirim C, et al. The influence of iloprost on acute lung injury induced by hind limb ischemia-reperfusion in rats. Pulm Pharmacol Ther 2005; 18: 235-41.

13. Grace PA. Ischemia-reperfusion injury. Br J Surg 1994; 81: 637.

14. Noiri E, Nakao A, Uchida K, Tsukahara H, Ohno M, Fujita T, et al. Oxidative and nitrosative stress in acute renal ischemia. Am J Physiol Renal Physiol 2001; 281: F948.

15. Versteilen AM, Di Maggio F, Leemreis JR, Groeneveld AB, Musters RJ, Sipkema P. Molecular mechanisms of acute renal failure following ischemia/reperfusion. Int J Artif Organs 2004; 27: 1019.

16. Lum H, Roebuck KA. Oxidant stress and endothelial cell dysfunction. Am J Physiol Cell Physiol 2001; 280: C719.

17. Bonventre JV, Weinberg JM. Recent advances in the pathophysiology of ischemic acute renal failure. J Am SocNephrol 2003; 14: 2199.

18. Legrand M, Ince C, Mik E, Johannes T, Payen D. Renal hypoxia and dysoxia following reperfusion of the ischemic kidney. Mol Med 2008; 14: 502-16.

19. Basile DP, Donohoe D, Roethe K, Osborn JL. Renal ischemic injury results in permanent damage to peritubular capillaries and influences long-term function. Am J Physiol Renal Physiol 2001; 281: F887-99.

20. Molitoris BA, Sandoval R, Sutton TA. Endothelial injury and dysfunction in ischemic acute renal failure. Crit Care Med 2002; 30: S235-40.

21. Brodsky SV, Yamamoto T, Tada T, Kim B, Chen J, Kajiya F, et al. Endothelial dysfunction in ischemic acute renal failure: rescue by transplanted endothelial cells. Am J Physiol Renal Physiol 2002; 282: F1140-9.

22. Sutton TA, Mang HE, Campos SB, Sandoval RM, Yoder MC, Molitoris BA. Injury of the renal microvascular endothelium alters barrier function after ischemia. Am J Physiol Renal Physiol 2003; 285: F191-8.

23. Goode HF, Webster NR, Howdle PD, Leek JP, Lodge JP, Sammi AS, et al. Reperfusion injury, antioxidants and hemodynamics during orthotopic liver transplantation. Hepatology 1994; 19: 354.

24. Xia Y, Khatchikian G, Zweier JY. Adenosin deaminase inhibition prevents free radical mediated injury in the postischemic heart. J Biol Chem 1996; 271: 10096.

25. Cherubini A, Polidori MC, Bregnocchi M, Pezzuto S, Cecchetti R, Ingegni $\mathrm{T}$, et al. Antioxidant profile and early outcome in stroke patients. Stroke 2000; 31: 2295.

26. Sadat U, Usman A, Gillard JH, Boyle JR. Does ascorbic acid protect against contrast induced- acute kidney injury in patients undergoing coronary angiography - a systematic review with meta-analysis of randomized controlled trials. J Am Coll Cardiol 2013; 62: 2167-75.

27. Zuurbier CJ, Emons VM, Ince C. Hemodynamics of anesthetized ventilated mouse models: aspects of anesthetics, fluid support, and strain. Am J Physiol Heart Circ Physiol 2002; 282: H2099-105.

28. Johannes T, Mik EG, Ince C. Dual-wavelength phosphorimetry for determination of cortical and subcortical microvascular oxygenation in rat kidney. J Appl Physiol 2006; 100: 1301-10.

29. Mik EG, Johannes T, Zuurbier CJ, Heinen A, Houben-Weerts JH, Balestra GM, et al. In vivo mitochondrial oxygen tension measured by a delayed fluorescence lifetime technique. Biophys J 2008; 95: 3977-90.

30. Bezemer R, Faber DJ, Almac E, Kalkman J, Legrand M, Heger M, et al. Evaluation of multi-exponential curve fitting analysis of oxygenquenched phosphorescence decay traces for recovering microvascular oxygen tension histograms. Med Biol Eng Comput 2010; 48: 1233-42.

31. Demirci C, Gargili A, Kandil A, Cetinkaya H, Uyaner I, Boynuegri B, et al. Inhibition of inducible nitric oxide synthase in murinevisceral larva migrans: effects on lung and liver damage. Chin J Physiol 2006;49: 326-34

32. O'Connor PM, Kett MM, Anderson WP, Evans RG. Renal medullary tissue oxygenation is dependent on both cortical and medullary blood flow. Am J Physiol Renal Physiol 2006; 290: F688-94.

33. Johannes T, Mik EG, Nohe B, Unertl KE, Ince C. Acute decrease in renal microvascular PO2 during acute normovolemichemodilution. Am J Physiol Renal Physiol 2007; 292: F796-803.

34. Cowley AW Jr, Mori T, Mattson D, Zou AP. Role of renal NO production in the regulation of medullary blood flow. Am J Physiol Regul Integr Comp Physiol 2003; 284: R1355-69.

35. Adler S, Huang H, Loke KE, Xu X, Tada H, Laumas A, et al. Endothelial nitric oxide synthase plays an essential role in regulation of renal oxygen consumption by NO. Am J Physiol Renal Physiol 2001; 280: F838 -43.

36. Laycock SK, Vogel T, Forfia PR, Tuzman J, Xu X, Ochoa M, et al. Role of nitric oxide in the control of renal oxygen consumption and the regulation of chemical work in the kidney. Circ Res 1998; 82: 1263-1271.

37. Karaman A, Turkmen E, Gursul C, Tas E, Fadillioglu E. Prevention of renal ischemia/reperfusion-induced injury in rats by leflunomide. Int J Urol 2006; 13: 1434-41.

38. Tyml K, Li F, Wilson JX. Delayed ascorbate bolus protects against maldistribution of microvascular blood flow in septic rat skeletal muscle. Crit Care Med 2005; 33: 1823-8.

39. Wang $\mathrm{P}, \mathrm{Zhu} \mathrm{Q}, \mathrm{Wu} \mathrm{N}$, Siow YL, Aukema HOK. Tyrosol attenuates ischemia-reperfusion-induced kidney injury via inhibition of inducible nitric oxide synthase. J Agric Food Chem 2013; 17; 61:366975. 
40. Szabó C, Módis K. Pathophysiological roles ofperoxynitritein circulatory shock. Shock 34: 4-14.

41. Gullans SR, Hebert SC. Metabolic basis of ion transport. In: Brenner and Rector's The Kidney (5th ed.), edited by Brenner BM. Philadelphia, PA: WB Saunders, 1996: 211-246.

42. Eschwege P, Paradis V, Conti M, Holstege A, Richet F, Devete J. In situ detection of lipid peroxidation by-products as markers of renal ischemia injuries in rat kidneys. J Urol 1999; 162: 553-7.

43. Sigh D, Chopra K. The effect of naringin, a bioflavonoid on ischemiareperfusion-induced renal injury in rats. Pharmacol Res 2004; 50: 187-193.

44. Baud L, Ardaillou R. Involvement of reactive oxygen species in kidney damage. Br Med Bull 1993; 49: 621-9.

45. Dreyer WJ, Smith CW, Michael LH, Rossen RD, Hughes BJ, Entman $\mathrm{ML}$, et al. Canine neutrophil activation by cardiac lymph obtained during reperfusion of ischemic myocardium. Circ Res 1989; 65: 175162.

46. Prescott SM, Zimmerman GA, Stafforini DM, McIntyre TM. Plateletactivating factor and related lipid mediators. Annu Rev Biochem 2000; 69: 419-45.

47. Lloberas N, Torras J, Herrero-Fresneda I, Cruzado JM, Riera M, Hurtado I, et al. Postischemic renal oxidative stress induces inflammatory response through PAF and oxidized phospholipids. Prevention by antioxidant treatment. FASEB J 2002; 16: 908-10.

How to cite this article: Ergin $B$, et al. Ascorbic acid improves renal microcirculatory oxygenation in a rat model of renal I/R injury. J Transl Intern Med 2015;3:116-125 